\title{
Evaluation of the Antimicrobial and Antibiofilm Effect of Chitosan Nanoparticles as Carrier for Supernatant of Mesenchymal Stem Cells on Multidrug-Resistant Vibrio cholerae
}

This article was published in the following Dove Press journal:

Infection and Drug Resistance

\section{Masoumeh Saberpour (iD \\ Bita Bakhshi (D) \\ Shahin Najar-peerayeh}

Department of Bacteriology, Faculty of Medical Sciences, Tarbiat Modares

University Tehran, Tehran, Iran
Correspondence: Bita Bakhshi Department of Bacteriology, Faculty of Medical Sciences, Tarbiat Modares University, Jalal-Ale-Ahmad Ave, Tehran |4||7-|3||6, Iran

$\mathrm{Tel} / \mathrm{Fax}+982182884558$

Email b.bakhshi@modares.ac.ir

\begin{abstract}
Aim: The aim of the present study was to evaluate the in vitro antimicrobial and antibiofilm activity of chitosan nanoparticles (CS NPs) incorporated with mesenchymal stem cellsderived conditioned media (MSCs CM) on MDR Vibrio cholerae strains.

Materials and Methods: Chitosan NPs were prepared and characterized by dynamic light scattering (DLS), scanning electron microscope (SEM) and zeta potential measurement. MSCs CM were prepared and entrapped into MSCs CM-CS NPs composite and its release efficiency was measured. Antibacterial efficacy of nano structures was determined by disk diffusion and broth microdilution methods. Antibiofilm activity was assessed by crystal violet assay.

Results: BM-MSCs were characterized to be negative for CD34 and CD45 markers, positive for CD73 and CD44 markers, and able to differentiate into osteoblast and adipocyte cells. The mean particle size of $96.6 \%$ of chitosan NPs was $414.9 \mathrm{~nm}$ with a suitable zeta potential and SEM morphology. Entrapment efficiency of MSCs CM-CS NPs was 76.9\%. Unstimulated MSCs CMCS NPs composite as a novel and proficient therapeutic nanostructure against MDR $V$. cholerae strains showed the synergistic activity of the two components of MSCs CM and CS NPs, leading to greater bacterial killing compared to control groups. MSCs CM more efficiently inhibited biofilm formation, although MSCs CM-CS NPs was also appeared to be effective in inhibiting biofilm formation compared to CS NPs and control group.

Conclusion: The designed nanodrug composite showed the best release in conditions mimicking the physiological conditions of the intestinal lumen. Given the fact that no overuse or genetic event would cause the emergence of antimicrobial resistance against the MSCs CM-CS NPs nanodrug, it could be considered as a promising alternative for the treatment of MDR $V$. cholerae infections in clinical settings.
\end{abstract}

Keywords: Vibrio cholerae, antimicrobial, chitosan nanoparticle, mesenchymal stem cells

\section{Introduction}

$V$. cholerae is still one of the most important causes of small intestine infections. Consumption of food and water contaminated with $V$. cholerae could cause symptoms of diarrhea in person. ${ }^{1,2}$ Most patients with this infection suffer from water loss and severe inflammation., ${ }^{3,4}$ Antibiotics along with electrolytes are prescribed for the treatment of patients with cholera. Antibiotics such as tetracyclines and macrolides are the first-line antibiotics for the treatment of diarrhea. ${ }^{5}$ Recently, antibiotics overuse has led to increased drug resistance among many bacteria, and increased antibiotic 
resistance has become one of the most important concerns in the world. Multidrug-resistant (MDR) $V$. cholerae strains have spread widely in several developing countries. ${ }^{6}$ There is a dramatic increase in the number of studies focusing on multidrug resistance phenomenon in bacteria.

Novel antibiotics are significantly produced, but the perpetual fear of the development of antibiotic resistance in bacteria has prompted researchers to focus on the probable application of non-antibiotic compounds as antimicrobial agents. Among the different antimicrobial agents under investigation, mesenchymal stem cells (MSCs) are supposed to tidily fulfill the critical criteria of a prevailing therapeutic agent. Recently, MSCs have been used as a novel therapeutic strategy for the treatment of bacterial diseases with inflammation. Many studies have suggested the protective role of MSCs against bacterial growth. ${ }^{7}$ A protective effect is induced by the release of soluble proteins from these cells, such as LL37, beta-defensin, interleukin-10 (IL-10), prostaglandin E2 (PGE2), tumor necrosis factor-alpha (TNF- $\alpha$ ), and IL- $6 .{ }^{8}$ Proteins secreted by MSCs have regulatory effects on the immune system. The use of MSCs secretions for the treatment of multidrugresistant Mycobacterium tuberculosis (MDR-TB) and Acinetobacter baumannii infections have been investigated and proved to be effective in vitro and in animal models. ${ }^{7,9}$ Moreover, the effect of antibiotics and MSCs have been evaluated individually and in combination, and their synergistic effect against multidrug-resistant Staphylococcus aureus strains has been demonstrated. ${ }^{10}$

Chitin is a polysaccharide with a fibrous structure. Chitin could be converted into chitosan by the removal of its acetyl group. Chitosan has been investigated as an antimicrobial compound against a wide range of microorganisms. Chitosan could show either bactericidal or bacteriostatic activity. It exhibits a variety of biological properties in medical fields. Properties such as biocompatibility, bioactivity, and nontoxicity have made chitosan as a very attractive compound in medical science. The potential application of this material is for the delivery of drugs such as painkillers and antimicrobial agents. ${ }^{11}$ Furthermore, chitosan nanoparticles have been used as carriers in vaccine development, they have also been shown to improve immune responses. ${ }^{12}$ Because chitosan nanoparticle has a receptor at $M$ and dendritic cells, this receptor effectively increases the entry of loaded material into the cell. ${ }^{13}$ Chitosan nanoparticles increase intestinal permeability and improve humoral and cellular immunity by increasing immune cells, resulting in direct bacterial killing. ${ }^{14}$
Biofilm is an aggregate of bacterial cells embedded in a matrix of extracellular polymeric substances (EPS) secreted by bacterial cells. This growth mode could be served as a defense mechanism against various environmental challenges and antibiotics. ${ }^{15}$ An antimicrobial agent is considered as effective in defending against bacterial infection if it could efficiently inhibit bacterial cells accumulation and biofilm development.

The previous studies have showed that chitosan is active against bacterial growth. ${ }^{16}$ However, no research has investigated the antimicrobial effects of MSCs conditioned media coupled with chitosan nanoparticles (MSCs CM-CS NPs) on MDR bacteria including $V$. cholerae, so far. In this study, it was hypothesized that incorporation of MSCs CM with CS NPs increases the antimicrobial efficacy via controlled release of cargo at the host-bacteria interface, this synergic effect along with the antimicrobial effects of these two components efficiently inhibit bacterial growth and improve disease. Therefore, the aim of the present study was to evaluate the in vitro antimicrobial and antibiofilm effect of CS NPs incorporated with MSCs CM on MDR $V$. cholerae strains.

\section{Materials and Methods}

\section{Bacterial Strains}

In the present study, multidrug-resistant $V$. cholerae strains were obtained from the archive of Tarbiat Modares University of Medical Science (Tehran, Iran). Antimicrobial susceptibility testing was performed for $V$. cholerae strains against five antibiotics. The strains showed resistance to tetracycline, ciprofloxacin, chloramphenicol, cotrimoxazole, and trimethoprim.

\section{Preparations and Characterization of Human Bone Marrow-Derived Mesenchymal Stem Cells (BM-MSC)}

BM-MSCs were purchased from Iranian Biological Resource Center (IBRC10094). The identity of MSCs was confirmed by evaluating the differentiation of these cells into osteoblasts and adipocytes using immunohistochemistry (IHC) method in our previous study. ${ }^{17}$ MSCs were evaluated using flow cytometry technique for CD34, CD45, CD44, and CD73 markers. ${ }^{17}$

\section{Cell Culture}

BM-MSCs were cultured and re-suspended in complete medium containing DMEM (Dulbecco's Modified Eagle's 
Medium) supplemented with 10\% FBS, 1\% penicillinstreptomycin, and $1 \%$ L-glutamine at $37^{\circ} \mathrm{C}$ with $5 \% \mathrm{CO}_{2}$. The medium was replaced once every $24-48 \mathrm{~h}$ for gradual purification of the cells. After reaching $80 \%$ confluency, the cells were treated with $0.5 \%$ trypsin (Gibco, USA) in order to be sub-cultured. MSCs were washed three times with phosphate-buffered saline (PBS) and transferred to a serum-free DMEM culture medium for $72 \mathrm{~h}$. MSCs CM were concentrated by centrifugation at $4000 \mathrm{rpm}$ for $30 \mathrm{~min}$ at $4^{\circ} \mathrm{C}$. Supernatants were obtained from bone marrow-derived MSCs (Passage 4). Concentrated MSCs CM were then sterilized on $0.22 \mu \mathrm{m}$ filters. MSCs CM were divided and placed into microtubes before freezing, and stored at $-80^{\circ} \mathrm{C}$ until use. $^{17}$

\section{Preparation of Nanoparticles}

For CS NPs preparation, $2 \mathrm{~mL}$ of sodium tripolyphosphate (TPP) was added to the PBS solution at a ratio of $1: 3$, then the prepared solution was added to $5 \mathrm{~mL}$ of chitosan solution at a ratio of 2:5 while stirring at room temperature. For MSCs CM-CS NPs composite preparation, MSCs CM were mixed with TPP (1:3); then $2 \mathrm{~mL}$ of MSCs CM-TPP was added to $5 \mathrm{~mL}$ of chitosan solution $(2: 5)$ at room temperature. The nanoparticle suspensions were stirred for $30 \mathrm{~min}$ and centrifuged at $16,000 \mathrm{rpm}$ for $30 \mathrm{~min}$. The resulting nanoparticle products were lyophilized and stored at $-4^{\circ} \mathrm{C}$ until use. ${ }^{18}$

\section{Characterization of Nanoparticles}

To characterize CS NPs, particles size was measured using dynamic light scattering (DLS) technique, particles charge was measured using a zeta potential measurement instrument, and particles shape was determined using a scanning electron microscope (SEM). ${ }^{19}$

\section{Evaluation of the Entrapment Efficiency (EE)}

Protein quantification was performed using the BCA assay kit at $572 \mathrm{~nm}$. The amount of protein in MSCs CM-CS NPs composite was determined indirectly by measuring the difference between the initial amount of protein dissolved in MSCs CM and the amount of protein remained in the supernatant after centrifugation. ${ }^{20}$

$$
\text { Entrapment efficiency }=\frac{\text { loaded protein in MSCs CM }-\mathrm{CSNp}}{\text { total amount of protein in MSCs CM }} \times 100
$$

\section{Release Assay}

The amount of protein released in the supernatant was measured using the BCA assay kit. Protein release was determined by dissolving protein-loaded nanoparticles in $1 \mathrm{~mL}$ of $10 \mathrm{mM}$ phosphate-buffered saline (PBS) at $\mathrm{pH}_{=} 7.2$ and 3.2 at $37^{\circ} \mathrm{C}$. MSCs CM-CS NPs solution was replaced with PBS at $1,2,3,4,24,48$, and 72 h time intervals. ${ }^{20}$

\section{Antibacterial Efficacy Assessment by Disk Diffusion Assay}

Antibacterial activity of MSCs CM-CS NPs was evaluated against $V$. cholerae strains using the disk diffusion method. The bacterium was cultured on Mueller-Hinton broth and incubated at $37{ }^{\circ} \mathrm{C}$ for $24 \mathrm{~h}$. Bacterial suspensions were prepared based on the standard 0.5 McFarland $\left(1.5 \times 10^{8}\right.$ $\mathrm{CFU} / \mathrm{mL}$ ). Stock cultures were streaked on the MuellerHinton agar plates. Furthermore, filter paper discs $(6 \mathrm{~mm}$ in diameter) were impregnated with each antimicrobial agent,

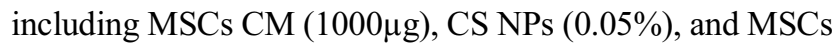
CM-CS NPs $(1000 \mu \mathrm{g}+0.05 \%)$. Caco- 2 cells supernatant was used as negative control. The plates were incubated at $37^{\circ} \mathrm{C}$ for $24 \mathrm{~h}$. Inhibition zones diameter was measured after 24 h. All inhibition assays were carried out in triplicate. ${ }^{21}$

\section{Antibacterial Efficacy Assessment by Microbroth Dilution Assay}

Antimicrobial activities of CS NPs, MSCs CM, and MSCs CM-CS NPs were examined by microdilution susceptibility testing using colony count method against $V$. cholerae strains. The microbroth dilution method was done according to the CLSI guidelines. The bacteria were grown to log phase in Mueller-Hinton broth at $37^{\circ} \mathrm{C}$ for $24 \mathrm{~h}$ to reach an optical density (OD) of 1:0 $\left(10^{8} \mathrm{CFU} / \mathrm{mL}\right)$. After $24 \mathrm{~h}$, a concentration of $5 \times 10^{5} \mathrm{CFU} / \mathrm{mL}$ of the bacteria was prepared. Microdilution technique was performed with $0.1 \mathrm{~mL}$

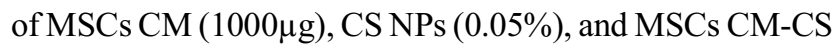
NPs $(1000 \mu \mathrm{g}+0.05 \%)$, respectively. About $0.1 \mathrm{~mL}$ of each compound was transferred to a 96-well plate. Each well was inoculated with $5 \times 10^{5} \mathrm{CFU} / \mathrm{mL}(0.01 \mathrm{~mL})$ of $V$. cholerae clinical strains and $0.1 \mathrm{~mL}$ of Mueller-Hinton broth and incubated at $37^{\circ} \mathrm{C}$ for $24 \mathrm{~h}$. Each sample was serially diluted to obtain a dilution of $10^{-5}$. Then $10 \mu \mathrm{L}$ of bacterial cells were cultured. After incubation, CFU of bacterial cells was calculated as follows: Number of colonies $\times 100 \times$ inverse dilution factor (M07, CLSI, 2019). 


\section{Antibiofilm Assay}

In this study, the antibiofilm effect of MSCs CM, CS NPs, and MSCs CM-CS NPs was evaluated by crystal violet assay in 96-well plates. Bacterial cells were grown in $1 \mathrm{~mL}$ BHI broth medium (Merck, Germany) for $24 \mathrm{~h}$ to reach an optical density (OD) of 1:0 (10 $\left.{ }^{8} \mathrm{CFU} / \mathrm{mL}\right)$. Bacterial suspensions were inoculated (1:100 dilution) into $1 \mathrm{~mL}$ BHI broth medium. Bacterial cultures were inoculated in the presence of MSCs CM, CS NPs, and MSCs CM-CS NPs for $24 \mathrm{~h}$ to allow biofilm formation.

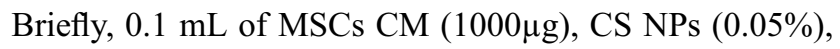
and MSCs CM-CS NPs $(1000 \mu \mathrm{g}+0.05 \%)$ were transferred to a 96-well plate containing $0.1 \mathrm{~mL}$ BHI broth supplemented with $0.5 \%(\mathrm{w} / \mathrm{v})$ sucrose, respectively. Then $0.01 \mathrm{~mL}$ of $V$. cholerae suspension $\left(10^{9} \mathrm{CFU} / \mathrm{mL}\right)$ was added into the 96-well plates and incubated for $24 \mathrm{~h}$ at $37^{\circ} \mathrm{C}$. Adherent cells were washed with distilled water and stained with $1 \%$ crystal violet (w/v in distilled water). The dye solution was de-stained with $95 \%$ ethanol for $45 \mathrm{~min}$, and OD of biofilm formation associated with crystal violet was defined at $570 \mathrm{~nm}$.

\section{Statistical Analysis}

Each assay in this study was carried out in triplicate. SPSS software Ver. 21.0 and GraphPad Prism Ver. 6 (by oneway ANOVA analysis) were used to perform statistical analyses. Results were expressed as the mean \pm standard deviation (SD), and $p$-value $<0.05$ was considered as statistically significant.

\section{Results}

\section{Characterization of Human Bone Marrow-Derived Mesenchymal Stem Cells}

BM-MSCs were negative for CD34 and CD45 markers and positive for CD73 and CD44 markers. The characterization results of bone marrow-derived MSCs indicated the differentiation of these cells into osteoblast and adipocyte cells. $^{17}$

\section{Characterization of Nanoparticles}

As shown in Table 1, the mean particle size of $96.6 \%$ of CS NPs was $414.9 \mathrm{~nm}$ (Figure 1A). The mean zeta potential of the synthesized CS NPs was $6.95 \mathrm{mV}$, they showed a suitable stability with $6.67 \mathrm{mV}$ zeta potential at $24.9^{\circ} \mathrm{C}$ and $1.2 \mathrm{mS} / \mathrm{cm}$ conductivity (Figure 1B).
Table I The Mean Particle Size and Zeta Potential of CS NPs

\begin{tabular}{|l|l|l|l|l|}
\hline Formula & $\begin{array}{l}\text { Chitosan } \\
\text { Concentration } \\
\%\end{array}$ & $\begin{array}{l}\text { Particle } \\
\text { Size (nm, } \\
\text { Mean } \pm \text { SD) }\end{array}$ & $\begin{array}{l}\text { Zeta } \\
\text { Potential } \\
(\mathbf{m V}, \text { Mean } \pm \\
\text { SD) }\end{array}$ & $\begin{array}{l}\text { EE\% } \\
\text { (Mean } \\
\pm \text { SD) }\end{array}$ \\
\hline CS NPs & 0.05 & $414.9 \pm 221.4$ & $6.95 \pm 10.3$ & $76.9 \%$ \\
\hline
\end{tabular}

\section{Scanning Electron Microscopy}

SEM technique was used to evaluate the surface morphology of the nanoparticles. The scanning electron micrographs of the chitosan nanoparticles showed that they were approximately spherical shape (Figure 2A). Although the surface of CS NPs was rough, the surface of MSCs CM-CS NPs was smooth. SEM imaging of the nanoparticles showed that in MSCs CM-CS NPs, the size dispersion was high, and the cross-linking between the molecules was very clear (Figure 2B).

\section{Entrapment Efficiency (EE \%)}

As shown in Table 1, EE of MSCs CM-CS NPs was 76.9\%. According to the EE results, a relatively high amount of MSCs CM was loaded onto the nanoparticles.

\section{In vitro Release Studies}

According to the release curves, MSCs CM was released from MSCs CM-CS NPs over a $72 \mathrm{~h}$ time period at $\mathrm{pH}=$ 7.2 and 3.2. In vitro protein release from MSCs CM-CS NPs was evaluated using phosphate buffer. At $\mathrm{pH}=7.2$, the release rate of MSCs CM from MSCs CM-CS NPs was $0 \%, 13 \%, 59 \%$, and $73 \%$ after $1,2,48$, and $72 \mathrm{~h}$, respectively. Also, after 2, 48, and $72 \mathrm{~h}$, the release rate of MSCs CM from MSCs CM-CS NPs was $0 \%, 29 \%$, and $43 \%$ at $\mathrm{PH}=3.2$, respectively. MSCs CM release rate was greater at $\mathrm{PH}=7.2$ than at $\mathrm{PH}=3.2$. Protein release rate was $73 \%$ and $43 \%$ at $\mathrm{PH}=7.2$ and 3.2 , respectively. Therefore, protein release rate from MSCs CM-CS NPs was reduced following the $\mathrm{pH}$ reduction (Figure 3).

\section{Antimicrobial Resistance/Susceptibility Testing of MDR V. cholerae Strains to Caco2 Supernatant, MSCs CM-CS NPs, CS NPs, and MSCs CM by Disk Diffusion Method}

According to the disk diffusion method results, the highest inhibitory effect was related to MSCs CM-CS NPs, followed by CS NPs (Table 2). The antimicrobial efficiency of MSCs CM-CS NPs was greater than that of other 


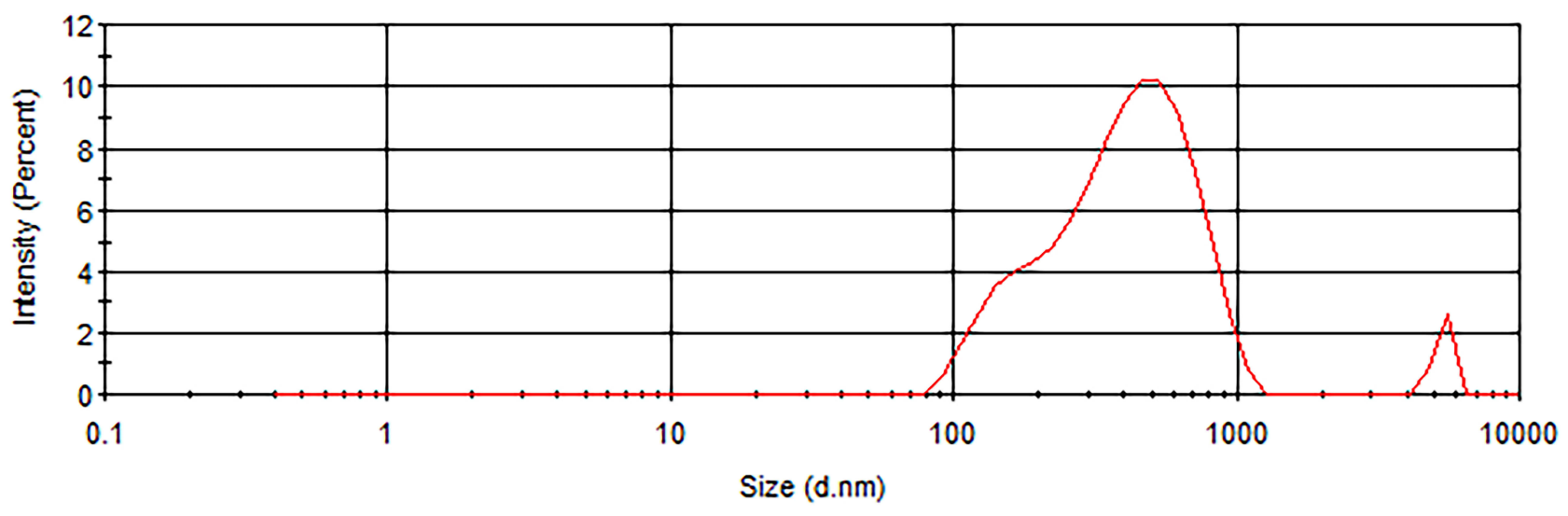

Record 1: NANo-Chi 1

B

\section{Zeta Potential Distribution}

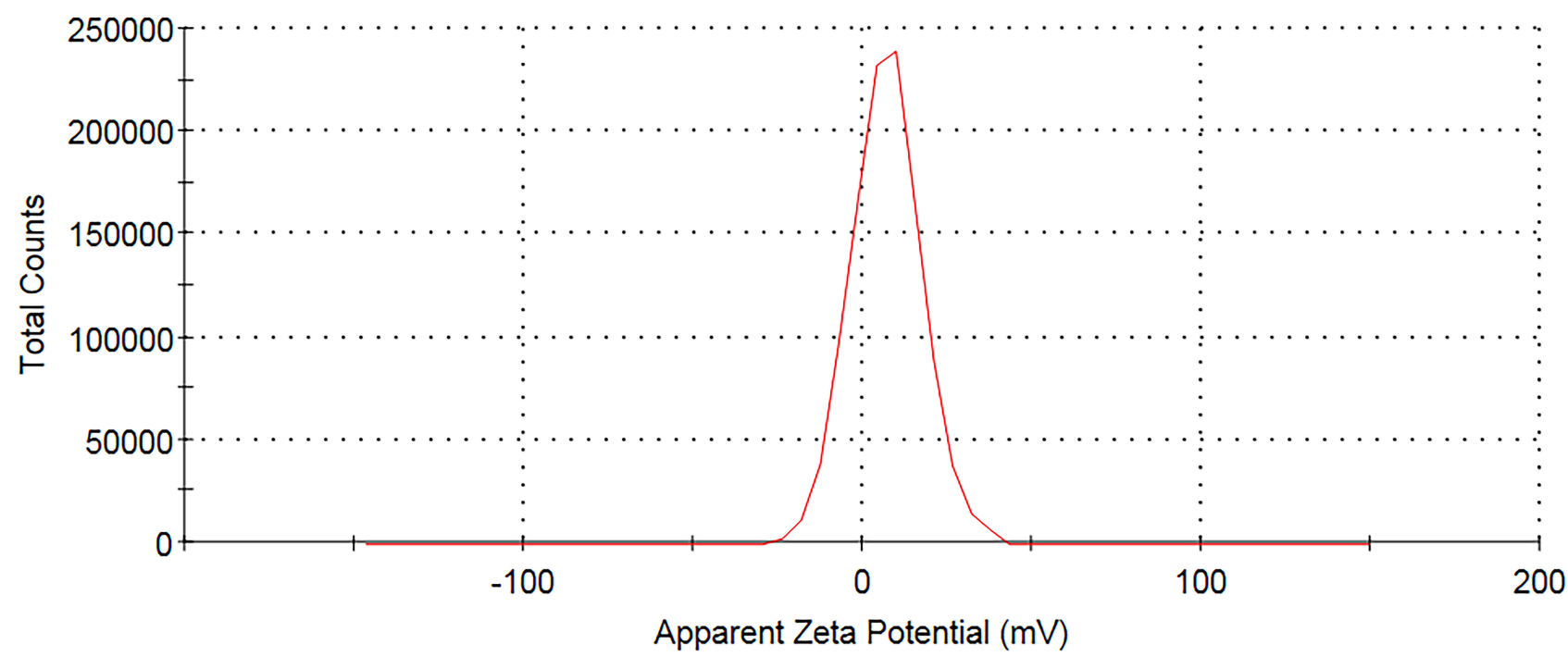

Figure I Zetasizer showed that the mean size of CS NPs was mostly in the range of 4 I4.9 nm (A). The zeta potential of CS NPs showed a good stability at $6.95 \mathrm{mV}(\mathbf{B})$.

groups. The measured inhibition zone diameter for $\mathrm{CS}$ NPs ranged between $22 \pm 0.4 \mathrm{~mm}$, for MSCs CM ranged between $15 \pm 0.1 \mathrm{~mm}$, and for MSCs CM-CS NPs ranged between $28 \pm 0.6 \mathrm{~mm}$ (Figure 4 ).

\section{Antimicrobial Effect of Three Different Compounds Against MDR V. cholerae Strains by CFU Count}

MSCs CM-CS NPs significantly inhibited bacterial growth compared to other groups. The antibacterial effect of all group was measured individually at similar concentrations.
The results showed that the antibacterial effects of MSCs CM at a concentration of $1000 \mu \mathrm{g}(0.1 \mathrm{~mL})$ and CS NPs at a concentration of $0.05 \%$ were not significant, separately; however, when combined with the same concentrations, the antibacterial effect of the prepared compound significantly increased. These results suggested the synergism between CS NPs and MSCs CM. The antibacterial effects of CS NPs and MSCs CM separately were observed at higher concentrations. In fact, MSCs CM and CS NPs individually inhibited bacterial growth at concentrations greater than $1000 \mu \mathrm{g}$ and $0.05 \%$ (Figure 5). 
A

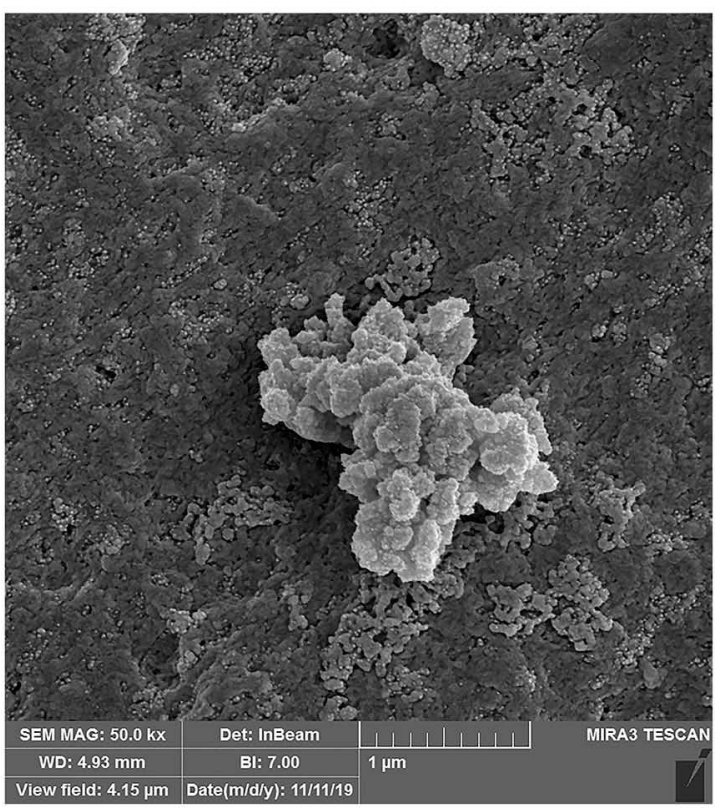

B

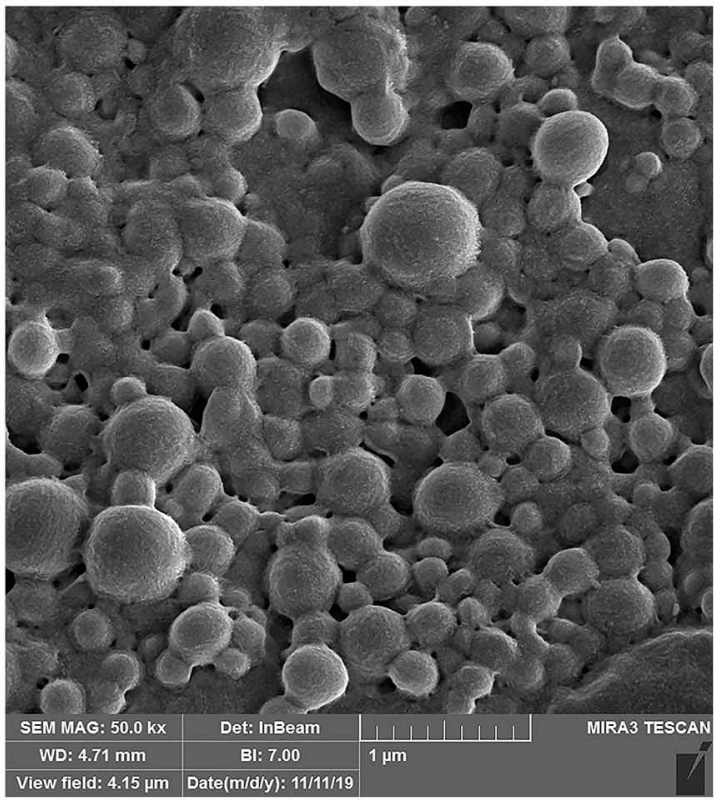

Figure 2 Physical properties of CS NPs and MSCs CM-CS NPs. SEM imaging of CS NPs (A). SEM imaging of MSCs CM-CS NPs (B).

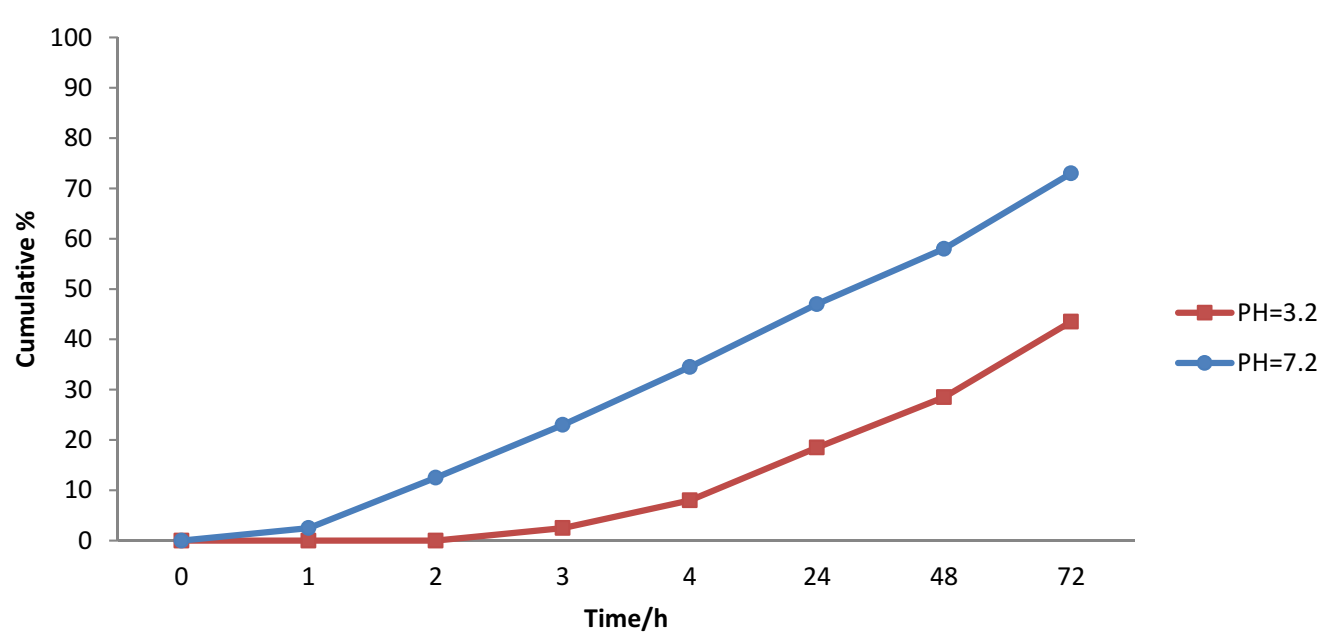

Figure 3 In vitro release of MSCs CM from MSCs CM-CS NPs at two different PH. Protein release rate in MSCs CM-CS NP increased at pH=7.2.

\section{Antibiofilm Activity of MSCs CM and MSCs CM-CS NPs Against MDR}

\section{$V$. cholerae Strains}

In vitro efficacy of different groups in reducing the biofilm formation capacity of MDR $V$. cholerae strains were evaluated. As shown in Figure 6, the antibiofilm effect of the following groups was assessed, including Group 1 (control group, PBS), Group 2 (MSCs CM $+V$. cholerae), Group 3 (V. cholerae), Group 4 (MSCs CM-CS NPs + V. cholerae), and Group 5 (V. cholerae + CS NPs) (Figure 6). Biofilm formation was measured photometrically at $\mathrm{OD}=570 \mathrm{~nm}$.
As shown in Figure 7, the antibiofilm activity of MSCs $\mathrm{CM}$ was higher than that of MSCs CM-CS NPs $(P<$ $0.0001)$. Indeed, the optical density (OD) of $0.547 \mathrm{~nm}$ was the mean of three replicates of Group 1 (PBS), and OD of $1.74 \mathrm{~nm}$ was the mean of three replicates of Group 3 ( $V$. cholerae). As shown in Table 3; biofilm formation was defined as non-adherent in Group 1 and $2(<0.547)$, weak in Group 4 (0.547-1.094), and intermediate in Group 3 and 5 (1.094-2.188), while in no group, it was defined as strong (>2.188). The results showed that CS NPs have no inhibitory role on biofilm formation. MSCs CM exhibited 
Table 2 Mean Inhibition Zone $(\mathrm{mm})$ of Four Tested Groups Against MDR V. cholerae Strains

\begin{tabular}{|l|l|l|l|l|}
\hline Species & $\begin{array}{l}\text { CS } \\
\text { NPs } \\
(\mathbf{m m}) \\
\pm \text { SD }\end{array}$ & $\begin{array}{l}\text { MSCs } \\
\text { CM } \\
(\mathbf{m m}) \pm \\
\text { SD }\end{array}$ & $\begin{array}{l}\text { MSCs CM- } \\
\text { CS NPs } \\
(\mathbf{m m}) \pm \text { SD }\end{array}$ & $\begin{array}{l}\text { Caco 2 } \\
\text { Supernatant } \\
(\mathbf{m m}) \pm \text { SD }\end{array}$ \\
\hline $\begin{array}{l}\text { MDR } \\
\text { V. cholerae }\end{array}$ & $22 \pm 0.4$ & $15 \pm 0.1$ & $28 \pm 0.6$ & 0 \\
\hline
\end{tabular}

a great inhibitory effect on the biofilm formation capacity of MDR $V$. cholerae strains, while MSCs CM-CS NPs appeared less efficient.

\section{Discussion}

Recently, MDR $V$. cholerae is increasing worldwide and has become a global public health concern. Excessive exposure of bacteria to antibiotics has altered bacterial genomes and led to the development of multidrug resistance in bacteria. ${ }^{6}$ Unfortunately, bacteria rapidly become resistant to antibiotics; therefore, researchers are progressively trying to develop new antibiotics. Considering the reasonable fear of consecutive emergence of antibiotic resistance, new non-antibiotic therapeutic strategies are needed to be developed in order for controlling diseases caused by MDR bacteria. MSCs-derived conditioned media are considered as an essential part of the innate immune system due to the presence of antimicrobial

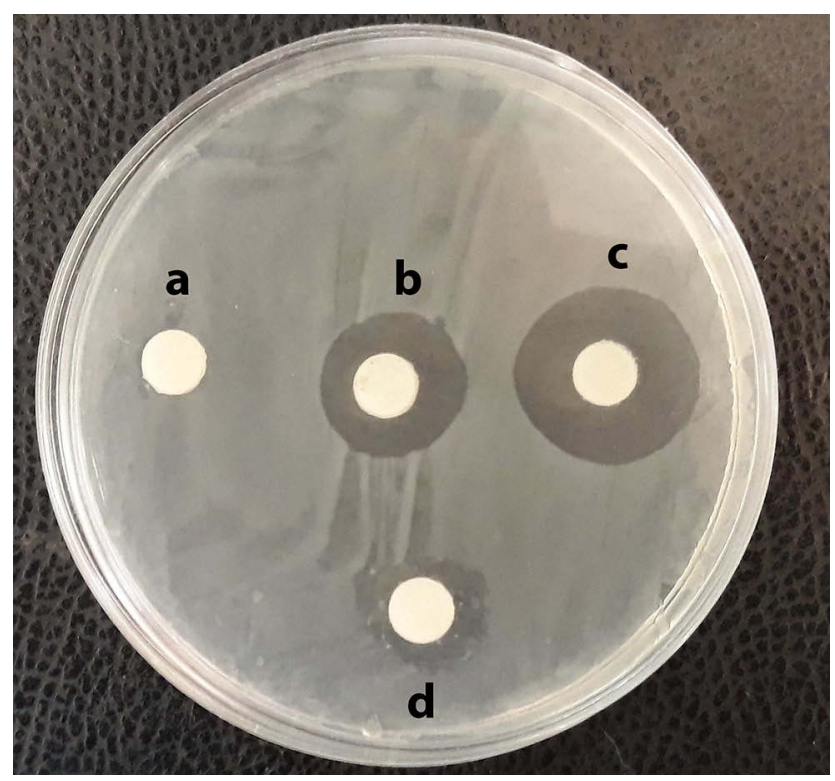

Figure 4 Mean inhibition zone $(\mathrm{mm})$ against MDR V. cholerae strains: Caco2 supernatant: $0 \mathrm{~mm}$ (A); CS NPs: $22 \mathrm{~mm}$ (B); MSCs CM-CS NPs: $28 \mathrm{~mm}$ (C); and MSCs CM: $15 \mathrm{~mm}$ (D).

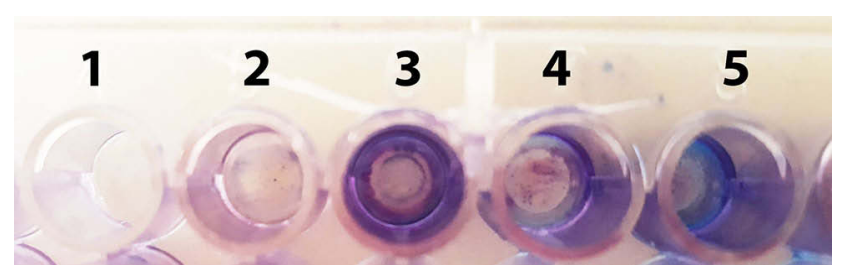

Figure 6 Antibiofilm effect of different groups against MDR $V$. cholerae strains, including: I) PBS; 2) MSCs CM + V. cholerae; 3) V. cholerae; 4) MSCs CM-CS NPs + V. cholerae; 5) V. cholerae + CS NP.

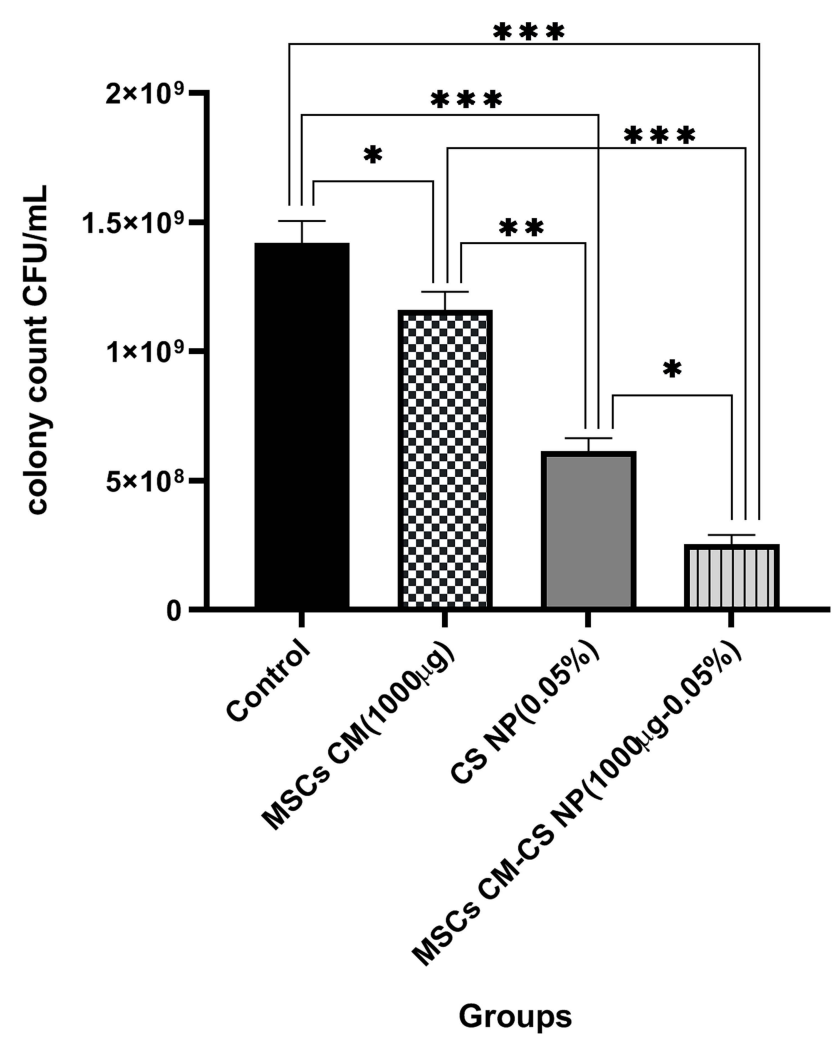

Figure 5 MSCs CM, CS NPs, and MSCs CM-CS NPs showed antimicrobial activity against MDR V. cholerae strains. Bacterial growth was assessed by CFU counts. Control group was $V$. cholerae strains alone. Bars are presented as mean of triplicate tests \pm SD. $* * * P<0.0008$ for control vs CS NPs; $* * * \mathrm{P}<0.0002$ for control vs MSC CM_CS NP; ***P< 0.0005 for MSCs CM vs MSCs CM-CS NPs; **P<0.0034 for MSCs CM vs CS NPs; *P<0.0158 for MSCs CM-CS NPs vs CS NPs; and *P<0.048 for control vs MSCs CM. Values showed the mean of triplicate tests \pm SD. Data were analyzed by ANOVA test; Bonferroni.

peptides, suggesting the antimicrobial activity of conditioned media against a wide range of bacteria. Previously, it was reported that unstimulated supernatant of bone marrow-derived mesenchymal stem cells consisted of soluble proteins and secreted vesicles, displaying marked antimicrobial activity against $V$. cholerae strains. The mechanism of antimicrobial activity of MSCs CM against $V$. cholerae strains is associated with the secreted products such as antimicrobial peptides. ${ }^{22}$ 


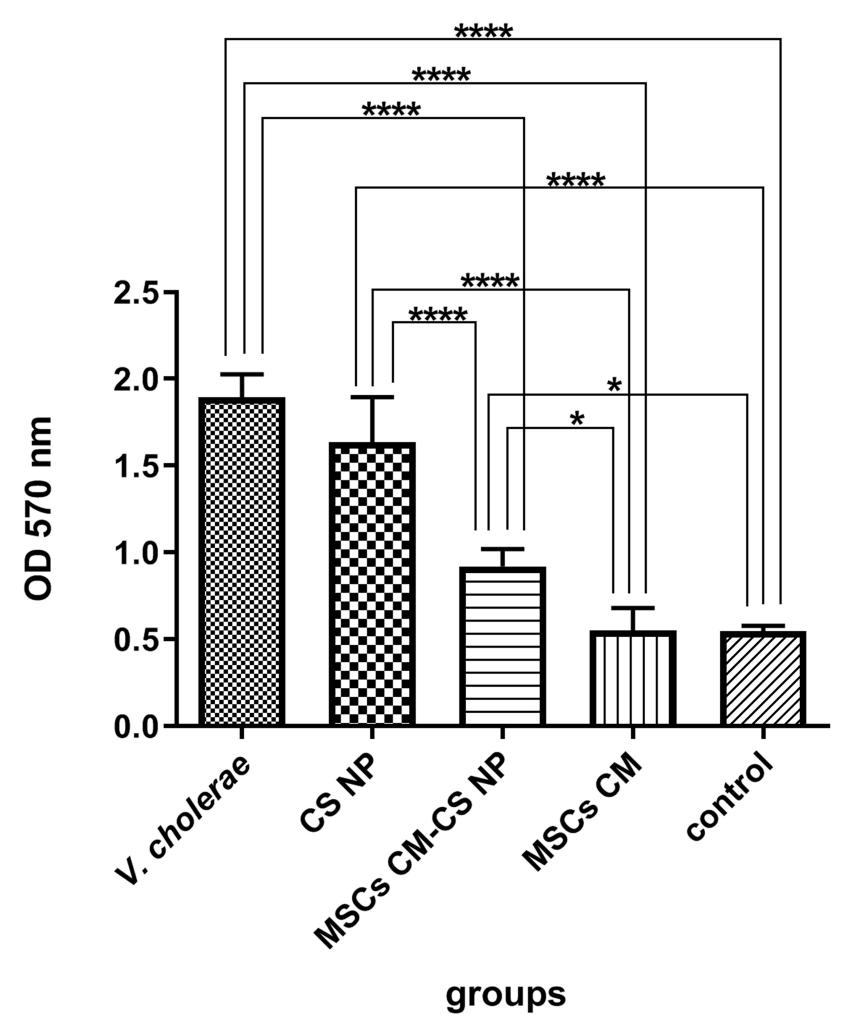

Figure 7 The inhibitory effects of different groups on biofilm formation of MDR $V$. cholerae strains. Bacterial suspension was incubated with each group. PBS was used as negative control. $* * * * P<0.0001$ for all groups vs $V$. cholerae and CS NPs; $* \mathrm{P}<0.02$ for MSCs CM-CS NPs vs control, and MSCs CM-CS NPs vs MSCs CM.

Chitosan is also a very suitable carrier with antibacterial activity. The exact mechanism of chitosan as an antimicrobial agent is not fully understood. Chitosan is positively charged and could therefore bind to the negatively charged bacterial cell membrane, resulting in the destruction of the membrane and leakage. ${ }^{21}$ Furthermore, $^{2}$ chitosan binds to LPS and act as a neutralizing agent. Moreover, chitosan promotes the inhibition of mRNA and protein synthesis through penetrating into the nuclei of the microorganisms. Also, it forms an external barrier chelating metals and nutrients which are essential for microbial growth. ${ }^{11}$ In previous studies, researchers have made great efforts to synthesize CS NPs with higher antibacterial activity and less toxicity. ${ }^{21}$ In this study, a lower concentration of CS NPs was used to make MSCs CM-CS NPs composite, and it was showed that incorporating lower concentrations of CS NPs with MSCs CM exhibited more inhibitory effect on MDR $V$. cholerae strains.

In a study conducted by Krasnodembskaya et al (2010), the expression of BM-MSCs antimicrobial peptides increased following the bacterial treatment. They demonstrated that to obtain higher antimicrobial effect mediated by soluble compounds such as LL37, MSCs should be induced with the bacteria. ${ }^{23}$ As mentioned earlier, uninduced MSCs CM could also reduce bacterial growth. One of the most important findings of the present study was that uninduced MSCs CM in combination with CS NPs reduced bacterial growth more efficiently. In fact, resistant bacteria to common antibiotics were sensitive against MSCs CM-CS NPs.

The present study findings revealed that combining MSCs CM with CS NPs is an effective method for inhibiting bacterial growth and could be considered as an adjunctive antimicrobial therapy for $V$. cholerae infections. The exact mechanism of this synergism is still unknown; however, previous studies have shown that some proteins secreted by MSCs, including LL37, have a disruptive effect on the cell wall and could play an important role in neutralizing LPS. ${ }^{24}$

Considering the mechanism of action of CS NPs on bacterial cell wall, it was proposed that probably this component makes bacterial cells more labile to the penetration of MSCs CM antimicrobial peptides and effectors, and since some proteins secreted by MSCs, including LL37, have a disruptive effect on the cell wall and could play an important role in neutralizing LPS, they enhance chitosan penetration into the bacterial cytoplasm and promote inhibition of mRNA and protein synthesis by chitosan.

$V$. cholerae strains form biofilms as a strategy to be able to persist in the environment. $V$. cholerae strains form biofilms during aquatic and intestinal phases of their life cycle. ${ }^{25}$ This growth mode enhances environmental persistence, provides protection against a number of environmental stresses, and increases antibiotic resistance in MDR $V$. cholerae

Table 3 Biofilm Formation of $V$. cholerae Strains Before and After the Exposure to Different Groups

\begin{tabular}{|l|l|l|l|}
\hline Groups & Mean of OD \pm SD & Biofilm Formation & Formula \\
\hline Group I: PBS & $0.547 \pm 0.02$ & Non adherent & OD<ODc \\
Group 2: MSCs CM + V. cholerae & $0.55 I \pm 0.1$ & Non adherent & OD<ODc, \\
Group 3: V. cholerae & $1.74 \pm 0.2$ & Intermediate & $2 O D c<O D<4 O D c$ \\
Group 4: MSCs CM-CS NP + V. cholerae & $0.91 \pm 0.08$ & Weak & ODc $<O D<2 O D c$ \\
Group 5: CS NP + V. cholerae & $1.79 \pm 0.2$ & Intermediate & $2 O D c<O D<4 O D c$ \\
\hline
\end{tabular}


strains. ${ }^{26}$ When $V$. cholerae strains enter the body, they must be physically protected against the acidic environment of the stomach. Biofilm network provides resistance to high acidity of the stomach environment. Eradication of biofilms is a serious global health concern in controlling bacterial infections because they cause microbial resistance to many antibiotics. ${ }^{27}$ Although non-antibiotic therapeutic strategies do not develop antimicrobial resistance, to be effective, they should be able to penetrate the biofilm structure or inhibit biofilm formation by bacterial population. For the first time in this study, the antibiofilm activity of MSCs CM and MSCs CM-CS NPs against MDR $V$. cholerae strains was assessed. It was showed that MSCs CM was able to more efficiently inhibit biofilm formation; although MSCs CM-CS NPs was also appeared to be effective in inhibiting biofilm formation compared to control group. Mechanism of MSCs CM in preventing biofilm formation may be due to the destruction of cell membrane or cell wall structure. However, further studies are needed to be conducted in order to discover the exact mechanisms involved.

Two different $\mathrm{pH}$ conditions (3.2 and 7.2) were chosen to investigate the release pattern of MSC CM from nanostructures, mimicking the acidic conditions of the gastrointestinal tract from mouth to intestinal lumen, and the best conditions for drug delivery was detected at $\mathrm{pH}=7.2$. It means that nanostructures do not release their cargo before reaching the target in the intestinal lumen, where they are supposed to interface with pathogen. Moreover, the MSCs CM-CS NPs composite exhibited a logical release pattern of MSCs CM with no burst releasing in the first few hours and with releasing approximately $40 \%$ of its cargo during $12-48 \mathrm{~h}$ at $\mathrm{pH}=7.2$, encompassing the time of its exposure to pathogen in the intestinal tract.

\section{Conclusion}

In conclusion, unstimulated MSCs CM-CS NPs as a novel and proficient therapeutic nanostructure against MDR $V$. cholerae strains greatly benefited from the synergistic activity of the two components. The designed nanodrug revealed high efficacy in antibiofilm activity which is considered as a crucial concern of antimicrobial agents in reaching the target. The nanodrug composite showed the best release in conditions mimicking the physiological conditions of the intestinal lumen. Given the fact that no overuse or genetic event would cause the emergence of antimicrobial resistance against the MSCs CM-CS NPs nanodrug, it could be considered as a promising alternative for the treatment of MDR $V$. cholerae infections in clinical settings.

\section{Ethics Statement}

The study was reviewed and approved by the Medical Ethics Committee of Tarbiat Modares University (Code: IR.MODARES.REC.1398.060) before it began and all research was performed in accordance with relevant guidelines/regulations.

\section{Data Sharing Statement}

The datasets of the current study are available within article or can be obtained from corresponding upon request.

\section{Acknowledgments}

This research was supported by research council of Tarbiat Modares University, Tehran, Iran. The authors would like to thank Mrs. Azita Bakhshi for critically editing the revised manuscript.

\section{Funding}

This work was supported by the Research Council of Tarbiat Modares University.

\section{Disclosure}

The authors declare that they have no competing interests or personal relationships that could have influenced the work reported in this paper.

\section{References}

1. Dashtbani-Roozbehani A, Bakhshi B, Katouli M, Pourshafie M. Comparative sequence analysis of recA gene among Vibrio cholerae isolates from Iran with globally reported sequences. Lett Appl Microbiol. 2011;53(3):313-323. doi:10.1111/j.1472-765X.2011.031 08. $\mathrm{x}$

2. Teh CSJ, Suhaili Z, Lim KT, et al. Outbreak-associated Vibrio cholerae genotypes with identical pulsotypes, Malaysia, 2009. Emerg Infect Dis. 2012;18(7):1177. doi:10.3201/eid1807.111656

3. Rezaie N, Pourshafie M. Increased resistance to tetracycline and erythromycin in vibrio cholerae clinical isolates isolated from patients with cholera disease during 2012-2013 outbreaks in IR Iran. Infect Epidemiol Microbiol. 2018;4(3):93-98.

4. Rezaie N, Bakhshi B, Najar-Peerayeh S. Distribution of resistance genetic determinants among Vibrio cholerae isolates of 2012 and 2013 outbreaks in IR Iran. Microb Pathog. 2017;104:12-16. doi:10.1016/j.micpath.2017.01.005

5. Sjölund-Karlsson M, Reimer A, Folster JP, et al. Drug-resistance mechanisms in Vibrio cholerae O1 outbreak strain, Haiti, 2010. Emerg Infect Dis. 2011;17(11):2151. doi:10.3201/eid1711.110720

6. Kitaoka M, Miyata ST, Unterweger D, Pukatzki S. Antibiotic resistance mechanisms of Vibrio cholerae. J Med Microbiol. 2011;60 (4):397-407. doi:10.1099/jmm.0.023051-0

7. Harman RM, Yang S, He MK, Van de Walle GR. Antimicrobial peptides secreted by equine mesenchymal stromal cells inhibit the growth of bacteria commonly found in skin wounds. Stem Cell Res Ther. 2017;8(1):157. doi:10.1186/s13287-017-0610-6 
8. Alcayaga-Miranda F, Cuenca J, Martin A, Contreras L, Figueroa FE, Khoury M. Combination therapy of menstrual derived mesenchymal stem cells and antibiotics ameliorates survival in sepsis. Stem Cell Res Ther. 2015;6(1):199. doi:10.1186/s13287-015-0192-0

9. Skrahin A, Jenkins HE, Hurevich H, et al. Effectiveness of a novel cellular therapy to treat multidrug-resistant tuberculosis. J Clin Tuberculosis Mycobacterial Dis. 2016;4:21-27. doi:10.1016/j. jctube.2016.05.003

10. Johnson V, Webb T, Norman A, et al. Activated mesenchymal stem cells interact with antibiotics and host innate immune responses to control chronic bacterial infections. Sci Rep. 2017;7(1):9575. doi:10.1038/s41598-017-08311-4

11. Goy RC, Britto D, Assis OB. A review of the antimicrobial activity of chitosan. Polimeros. 2009;19(3):241-247. doi:10.1590/S010414282009000300013

12. Tabrizi NM, Amani J, Ebrahimzadeh M, Nazarian S, Kazemi R, Almasian P. Preparation and evaluation of chitosan nanoparticles containing CtxB antigen against Vibrio cholera. Microb Pathog. 2018;124:170-177. doi:10.1016/j.micpath.2018.08.037

13. Fasihi-Ramandi M, Ghobadi-Ghadikolaee H, Ahmadi-Renani S, Taheri RA, Ahmadi K. Vibrio cholerae lipopolysaccharide loaded chitosan nanoparticle could save life by induction of specific immunoglobulin isotype. Artif Cells Nanomed Biotechnol. 2018;46 (1):56-61. doi:10.1080/21691401.2017.1290646

14. Marandi BHG, Zolfaghari MR, Kazemi R, Motamedi MJ, Amani J. Immunization against Vibrio cholerae, ETEC, and EHEC with chitosan nanoparticle containing LSC chimeric protein. Microb Pathog. 2019;134:103600. doi:10.1016/j.micpath.2019.103600

15. Bhattacharya SP, Bhattacharya A, Sen A. A comprehensive and comparative study on the action of pentacyclic triterpenoids on Vibrio cholerae biofilms. bioRxiv. 2020.

16. Costa EM, Silva S, Vicente S, Veiga M, Tavaria F, Pintado M. Chitosan as an effective inhibitor of multidrug resistant Acinetobacter baumannii. Carbohydr Polym. 2017;178:347-351. doi:10.1016/j.carbpol.2017.09.055

17. Bahroudi M, Bakhshi B, Soudi S, Najar-peerayeh S. Antibacterial and antibiofilm activity of bone marrow-derived human mesenchymal stem cells secretome against Vibrio cholerae. Microb Pathog. 2020;139:103867. doi:10.1016/j.micpath.2019.103867
18. Piras AM, Maisetta G, Sandreschi S, et al. Chitosan nanoparticles loaded with the antimicrobial peptide temporin B exert a long-term antibacterial activity in vitro against clinical isolates of Staphylococcus epidermidis. Front Microbiol. 2015;6:372. doi:10. 3389/fmicb.2015.00372

19. Jeong Y, Lim DW, Choi J. Assessment of size-dependent antimicrobial and cytotoxic properties of silver nanoparticles. Adv Mater Sci Eng. 2014;2014.

20. Almaaytah A, Mohammed GK, Abualhaijaa A, Al-Balas Q. Development of novel ultrashort antimicrobial peptide nanoparticles with potent antimicrobial and antibiofilm activities against multidrug-resistant bacteria. Drug Des Devel Ther. 2017;11:3159. doi:10.2147/DDDT.S147450

21. Tanha N, Karimzadeh K, Zahmatkesh A. A study on the antimicrobial activities of chitin and chitosan extracted from freshwater prawn shells (Macrobrachium nipponense). Int J Health Stud. 2017;3(3).

22. Driscoll J, Patel T. The mesenchymal stem cell secretome as an acellular regenerative therapy for liver disease. $J$ Gastroenterol. 2019;1-11.

23. Krasnodembskaya A, Song Y, Fang X, et al. Antibacterial effect of human mesenchymal stem cells is mediated in part from secretion of the antimicrobial peptide LL-37. Stem Cells. 2010;28(12):2229-2238. doi:10.1002/stem.544

24. Scott A, Weldon S, Buchanan PJ, et al. Evaluation of the ability of LL-37 to neutralise LPS in vitro and ex vivo. PLoS One. 2011;6(10): e26525. doi:10.1371/journal.pone.0026525

25. Teschler JK, Zamorano-Sánchez D, Utada AS, et al. Living in the matrix: assembly and control of Vibrio cholerae biofilms. Nat Rev Microbiol. 2015;13(5):255-268. doi:10.1038/nrmicro3433

26. Meza-Villezcas A, Gallego-Hernández AL, Yildiz FH, Jaime-Acuña OE, Raymond-Herrera O, Huerta-Saquero A. Effect of antimicrobial nanocomposites on Vibrio cholerae lifestyles: pellicle biofilm, planktonic and surface-attached biofilm. PLoS One. 2019;14(6):e0217869. doi:10.1371/journal.pone.0217869

27. Pederson DB, Dong Y, Blue LB, Smith SV, Cao M. Water-soluble cranberry extract inhibits Vibrio cholerae biofilm formation possibly through modulating the second messenger 3', 5'-Cyclic diguanylate level. PLoS One. 2018;13(11):e0207056. doi:10.1371/journal. pone.0207056
Infection and Drug Resistance

\section{Publish your work in this journal}

Infection and Drug Resistance is an international, peer-reviewed openaccess journal that focuses on the optimal treatment of infection (bacterial, fungal and viral) and the development and institution of preventive strategies to minimize the development and spread of resistance. The journal is specifically concerned with the epidemiology of antibiotic resistance and the mechanisms of resistance development and diffusion in both hospitals and the community. The manuscript management system is completely online and includes a very quick and fair peerreview system, which is all easy to use. Visit http://www.dovepress.com/ testimonials.php to read real quotes from published authors. 